\title{
Sinopse Taxonômica de Oxalidaceae no Estado de Alagoas, Brasil
}

\author{
Ykaro Richard Oliveira ${ }^{1,3} \mathrm{e}$ (1D) Maria Carolina de Abreu ${ }^{2}$
}

Recebido: 08.08.2019; aceito: 21.01.2020

Como citar: Oliveira, Y.R. \& Abreu, M.C. 2020. Sinopse Taxonômica de Oxalidaceae no Estado de Alagoas, Brasil. Hoehnea 47: e842019. http://dx.doi.org/10.1590/2236-8906-84/2019

ABSTRACT - (Taxonomic synopsis of Oxalidaceae in Alagoas State, Brazil). This work consists of a synoptic treatment for Oxalidaceae family in Alagoas State, Northeast Brazil. Two genera and 12 species were found: Averrhoa with one species and Oxalis with 11 species. Oxalis neuwiedii Zucc. and O. triangularis A. St.-Hil. are recorded for the first time in the Alagoas State. Taxonomic keys for species recognition are provided, as well, illustrations and distribution data.

Keywords: Averrhoa, geographical distribution, Northeast, Oxalis, taxonomy

RESUMO - (Sinopse taxonômica de Oxalidaceae no Estado de Alagoas, Brasil). Este trabalho consiste no tratamento sinóptico da família Oxalidaceae no Estado de Alagoas, Nordeste do Brasil. Foram encontrados dois gêneros e 12 espécies: Averrhoa com uma espécie e Oxalis representado por 11 espécies. Oxalis neuwiedii Zucc. e O. triangularis A. St.-Hil. são registradas pela primeira vez para o Estado de Alagoas. São fornecidas chaves taxonômicas para o reconhecimento das espécies, bem como ilustrações e dados de distribuição.

Palavras-chave: Averrhoa, distribuição geográfica, Nordeste, Oxalis, taxonomia

\section{Introdução}

Oxalidaceae R. Br. pertence à ordem Oxalidales (APG IV 2016) e compreende cinco gêneros e cerca de 880 espécies que se distribuem nas regiões tropicais e temperadas do globo (Cocucci 2004), cujos principais centros de dispersão são a América do Sul e África austral (Oberlander et al. 2011). É característico da família o hábito variado entre ervas, subarbustos, arbustos ou árvores; folhas tri ou plurifolioladas (raramente unifolioladas); flores actinomorfas, diclamídeas e bissexuais e fruto do tipo cápsula loculicida ou baga. A presença de heterostilia também é marcante na família, sendo seus representantes principalmente alógamos (Lourteig 1983, Judd et al. 1999).

No Brasil são registradas aproximadamente 103 espécies e três gêneros de Oxalidaceae: Averrhoa L., Biophytum DC. e Oxalis L. (Abreu \& Fiaschi 2015). Averrhoa tem ocorrência subespontânea (Souza \& Lorenzi 2008), Biophytum tem sua distribuição concentrada na Amazônia e na Mata Atlântica e Oxalis ocorre em todo o país (Lourteig 1994). Oxalis é o gênero mais representativo e possui cerca de 500 espécies
(Abreu \& Fiaschi 2009), das quais 97 ocorrem no Brasil (Abreu \& Fiaschi 2015). Os representantes desse gênero são encontrados em diversos ambientes, principalmente em regiões abertas e áreas antropizadas (Fiaschi \& Conceição 2005).

Segundo Grigoletto et al. (2014), no Brasil não há uma pesquisa detalhada acerca da taxonomia de Oxalis, contudo, destacam-se alguns estudos florísticos regionais abordando a família, como os trabalhos envolvendo Oxalidaceae na Floresta Atlântica, no Parque Estadual das Fontes de Ipiranga, Estado de São Paulo, das Cangas da Serra dos Carajás, Estado do Pará, na Serra do Cipó e na região de Grão-Mogol, Estado de Minas Gerais e nos Estados do Ceará, Distrito Federal, Goiás, Mato Grosso do Sul, Paraná, Pernambuco, São Paulo, Santa Catarina, Rio de Janeiro, Rio Grande do Sul e Tocantins (Abreu et al. 2008, 2013, Abreu \& Fiaschi 2009, Conceição \& Giulietti 1998, 2004, Costa et al. 2019, Fiaschi 2006, 2014, 2018, Fiaschi \& Conceição 2005, Grigoletto et al. 2014, Hall et al. 2017, Loiola et al. 2018, Lourteig 1983, Souza \& Bianchini 2000).

1. Unidade Escolar Ozildo Albano, Rua Marcos Parente, Centro, 64600-000 Picos, PI, Brasil

2. Universidade Federal do Piauí, Laboratório de Botânica, Campus Senador Helvídio Nunes de Barros, Rua Cícero Duarte, 905 , Bairro Junco, 64.607-670 Picos, PI, Brasil

3. Autor para correspondência: ykr-oliveira@hotmail.com 
O presente trabalho teve como objetivo verificar a diversidade e distribuição, assim como fornecer uma chave taxonômica e ilustrações para o reconhecimento dos gêneros e espécies da família Oxalidaceae encontradas no Estado de Alagoas, contribuindo para o inventário da flora fanerogâmica do Estado.

\section{Materiais e métodos}

Área de estudo - Com uma área de 27.767,661 Km², o Estado de Alagoas encontra-se na porção oriental do Nordeste brasileiro e limita-se ao norte com o Estado de Pernambuco, nos meandros do Rio Jacuípe; ao sul com o Estado do Sergipe, separados pelo Rio São Francisco; ao oeste com o Estado da Bahia e ao leste com o Oceano Atlântico. A temperatura média anual ultrapassa $24{ }^{\circ} \mathrm{C}$ e os índices pluviométricos são inferiores a $1.000 \mathrm{~mm}$ no semiárido do Estado (IMA 2005, Lyra-Lemos et al. 2010). Quanto à formação vegetacional, o Estado de Alagoas apresenta um conjunto florístico-vegetacional ainda pouco conhecido (Melo \& Lyra-Lemos 2008), que se encontra fitogeograficamente representado pelos domínios Caatinga e Mata Atlântica.

Tratamento taxonômico - A análise morfológica foi baseada em espécimes pertencentes à coleção MAC, complementadas pela análise de materiais dos herbários ESA, HUEFS, IPA, PEUFR, RB e SP listados conforme Thiers et al. (2019) (constantemente atualizado) e do Herbário Sérgio Tavares (HST) da Universidade Federal Rural do Pernambuco, Recife, PE, Brasil. A identificação dos espécimes foi baseada em Lourteig $(1975,1979,1980$, 1982, 1983, 1994, 2000) e Progel (1877). A padronização da terminologia das estruturas vegetativas e reprodutivas baseou-se em Radford et al. (1974) e Harris \& Harris (1994).
A grafia dos nomes dos autores foi verificada em Brummitt \& Powell (1992) e as abreviaturas das obras princeps em Stafleu \& Cowan (1976-1988). Dados acerca da distribuição geográfica foram obtidos a partir das etiquetas presentes nos materiais depositados nos herbários; e os mapas de distribuição das espécies foram confeccionados por meio do programa QGIS.

\section{Resultados e discussão}

Oxalidaceae R. Br., Narr. Exp. Zaire: 433. 1818.

Árvores (Averrhoa), arbustos ou mais comumente ervas; quando ervas, bulbosas ou caulescentes, eretas ou prostradas. Folhas alternas, subopostas, pseudoverticiladas, compostas trifolioladas ou pinadas (Averrhoa); estípulas presentes ou não. Inflorescências cimosas, 1-2-fidas, plurifloras ou unifloras. Flores geralmente vistosas, actinomorfas, diclamídeas, pentâmeras; sépalas 5, imbricadas, verdes ou vináceas; pétalas 5 , livres na base, conadas na porção mediana, imbricadas, uniformemente amarelas, roxas, lilases ou róseas ou com coloração variando de tom entre a fauce e o lobo; estames 10 , dispostos em dois ciclos de tamanhos distintos, os maiores alternipétalos ligulados ou não, os menores opositipétalos; anteras bitecas e rimosas; carpelo único, ovário súpero, geralmente pentalobado, estiletes 5, livres, terminais, estigmas curtamente bífidos, lobados ou capitados. Fruto baga pentalobada (Averrhoa) ou cápsula com cálice $\mathrm{e}$ estiletes persistentes, deiscência loculicida. Sementes com testa ornamentada.

No Estado de Alagoas foram encontrados dois gêneros e 12 espécies: Averrhoa com uma espécie e Oxalis com 11 espécies, que são tratadas a seguir.

Chave de identificação das espécies de Oxalidaceae no Estado de Alagoas

1. Folhas com mais de quatro folíolos, fruto baga.

1. Averrhoa carambola

1. Folhas sempre trifolioladas, fruto cápsula

2. Flores com lobos da corola alvos, róseos ou lilases

3. Erva bulbosa, lâminas foliares triangulares, cápsula cilíndrica ou fusiforme

11. Oxalis triangularis

3. Erva não bulbosa, lâminas foliares elípticas, ovadas ou lanceoladas, cápsula oblonga ou subglobosa

4. Folhas alternas ou subopostas

3. Oxalis cratensis

4. Folhas pseudoverticiladas 8. Oxalis neuwiedii

2. Flores com lobos da corola amarelos

5. Ervas prostradas

6. Folhas pinadas, cápsula globoso-achatada 6. Oxalis glaucescens

6. Folhas digitadas, cápsula cilíndrico-elipsoide 3. Oxalis corniculata

5. Ervas eretas, subarbustos ou arbustos

7. Folhas pseudoverticiladas

8. Braquiblastos presentes, cápsula oblonga 5. Oxalis frutescens 8. Braquiblastos ausentes, cápsula subglobosa 12. Oxalis umbraticola 


\section{Folhas alternas ou subopostas}

9. Folíolos com lâminas glabras ou glabrescentes em ambas as faces

10. Ramos glabros, folíolos com lâminas ovadas a elípticas, sépalas elípticas, estigmas capitados, cápsulas oblongas 10. Oxalis sepium

10. Ramos híspidos-glandulares, folíolos com lâminas estreitamente oblongas a obovadas, sépalas ovadas, estigmas bilobados, cápsulas globosas.

4. Oxalis divaricata

9. Folíolos com lâminas tomentosas ou seríceas na face abaxial

11. Lâminas com ápice agudo, cápsulas oblongas com três sementes por lóculo 7. Oxalis hedysarifolia

11. Lâminas com ápice acuminado, cápsulas ovoide-globosas com uma semente por lóculo

9. Oxalis psoraleoides

1. Averrhoa carambola L., Sp. Pl. 1: 428. 1753.

Figuras 1, 2 b, 4 d-e

Espécie cultivada, originária da Indonésia e Malásia, introduzida no Brasil onde é amplamente distribuída, entretanto, por ser uma espécie cultivada, usualmente não é descrita em trabalhos que abordam a florística de Oxalidaceae (Fiaschi \& Conceição 2005, Grigoletto et al. 2014, Loiola et al. 2018). Dentre as espécies de Oxalidaceae registradas para o Estado de Alagoas, Averrhoa carambola é única que possui folhas com mais de quatro folíolos e fruto do tipo baga alongada, que em seção transversal possui formato estrelado. Esta espécie destaca-se ainda pelo seu potencial econômico por possuir frutos comestíveis (Donadio et al. 2001). No Estado de Alagoas foi coletada em áreas urbanizadas dos municípios de Arapiraca, Belém e Maceió.

Material examinado selecionado: BRASIL. ALAGOAS: Arapiraca, Povoado Cangaçu, 13-I-2012, fl. L.S. Pereira \& A.C.S. Oliveira s.n. (MAC54386); Belém, Sítio Cabeça
Dantas a $5 \mathrm{Km}$ da cidade, 11-XI-1993, fl. C.S.S. Barros 132 (MAC); Maceió, Bairro do Canaã, 16-II-2012, fl. e fr., T.M.G. Cerqueira 26 (MAC).

2. Oxalis corniculata L., Sp. Pl. 1: 435. 1753.

Figuras $1,4 \mathrm{j}$

Esta espécie é cosmopolita provavelmente originária do sudeste da Ásia (Groom et al. 2019) e está amplamente distribuída na América do Sul, sendo frequente em ambientes antropizados e jardins (Lourteig 2000) e, por ser uma espécie ruderal, é pouco coletada (Fiaschi \& Conceição 2005). Distingue-se das demais espécies encontradas no Estado de Alagoas por ser uma erva prostrada com folhas digitadas e cápsulas cilíndrico-elipsoides. No Estado é encontrada em Caatinga e Mata Pluvial Atlântica (tabela 1). Espécimes com flores e frutos foram coletados em janeiro, abril e agosto.

Material examinado selecionado: BRASIL. ALAGOAS: Arapiraca, Mangabeiras, 22-I-2010, fl. e fr., E.C.O. Chagas

Tabela 1. Ocorrência das espécies de Oxalidaceae no Estado de Alagoas, Brasil, pelo tipo de vegetação.

Table 1. Occurrence of Oxalidaceae species in Alagoas State, Brazil, by vegetation type.

\begin{tabular}{|c|c|c|}
\hline Táxon & Domínio fitogeográfico & Vegetação \\
\hline Oxalis corniculata $\mathrm{L}$. & Caatinga; Mata Atlântica & Caatinga; Floresta ombrófila \\
\hline Oxalis cratensis Hook. & Mata Atlântica & $\begin{array}{l}\text { Floresta ciliar; Floresta estacional semidecidual; } \\
\text { Floresta ombrófila }\end{array}$ \\
\hline Oxalis divaricata Mart. ex Zucc. & Caatinga; Mata Atlântica & Caatinga; Floresta estacional semidecidual; Restinga \\
\hline Oxalis frutescens $\mathrm{L}$. & Caatinga & Caatinga \\
\hline Oxalis glaucescens Norlind & Caatinga & Caatinga; Mata estacional decidual \\
\hline Oxalis hedysarifolia Raddi & Caatinga; Mata Atlântica & $\begin{array}{l}\text { Caatinga; Campo rupestre; Floresta estacional } \\
\text { semidecidual; Floresta ombrófila; Vegetação } \\
\text { antropizada; Vegetação sobre afloramentos rochosos }\end{array}$ \\
\hline Oxalis neuwiedii Zucc. & Mata Atlântica & $\begin{array}{l}\text { Floresta estacional semidecidual; Floresta ombrófila; } \\
\text { Vegetação antropizada }\end{array}$ \\
\hline Oxalis psoraleoides Kunth & Caatinga; Mata Atlântica & Caatinga; Estepe; Floresta estacional semidecidual \\
\hline Oxalis sepium A. St.-Hil. & Caatinga & Caatinga; Floresta estacional semidecidual \\
\hline Oxalis triangularis A. St.-Hil. & Mata Atlântica & Floresta ombrófila \\
\hline Oxalis umbraticola A. St.-Hil. & Mata Atlântica & Floresta ombrófila \\
\hline
\end{tabular}




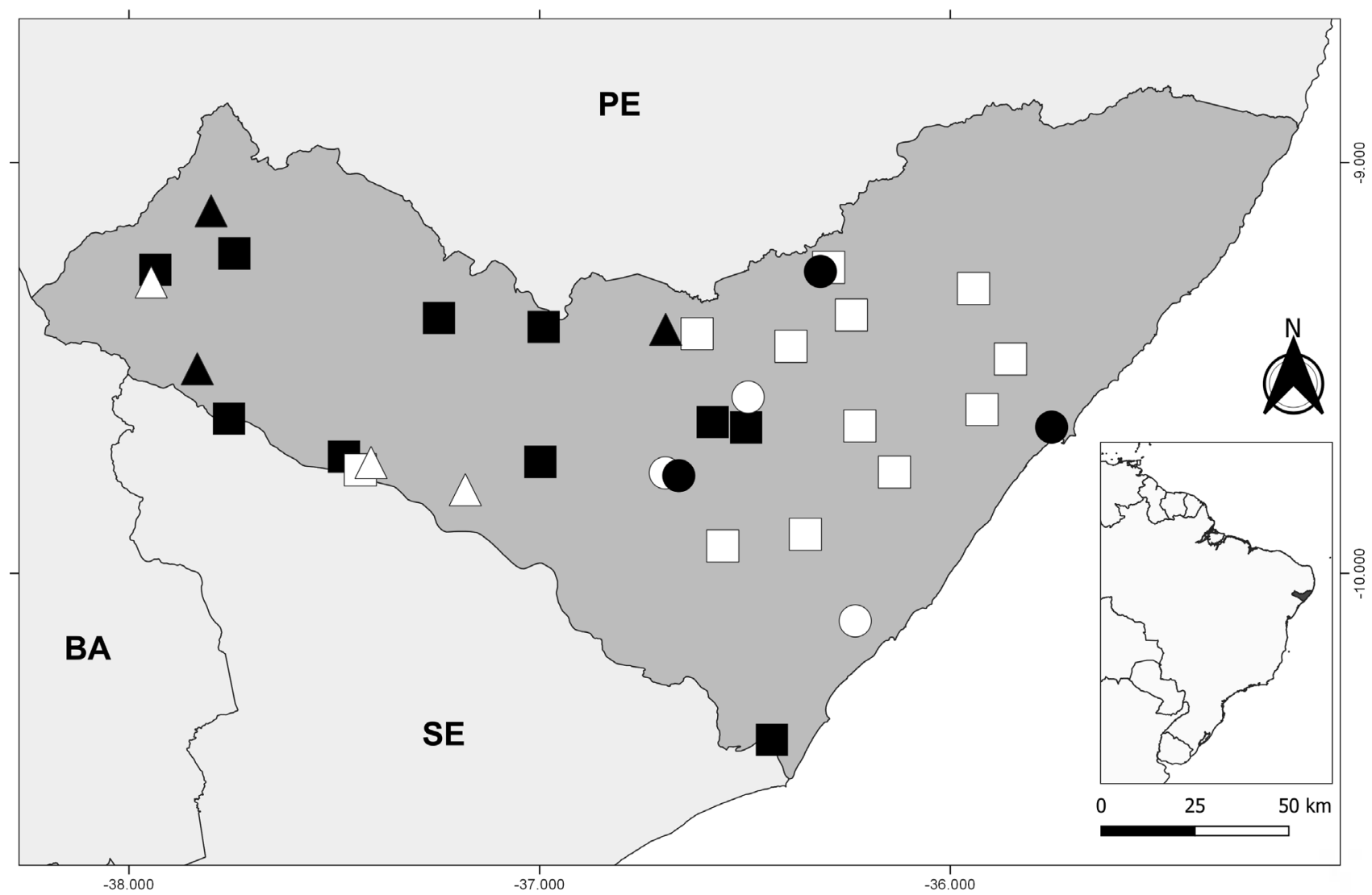

Figura 1. Distribuição das espécies no Estado de Alagoas, Brasil. Averrhoa carambola L. (círculos brancos), Oxalis corniculata L. (círculos pretos), Oxalis cratensis Hook. (quadrados brancos), Oxalis divaricata Mart. ex Zucc. (quadrados pretos), Oxalis frutescens L. (triângulos pretos) e Oxalis glaucescens Norlind (triângulos brancos). BA: Estado da Bahia. PE: Estado de Pernambuco. SE: Estado de Sergipe.

Figure 1. Distribution of species in Alagoas State, Brazil: Averrhoa carambola L. (white circles), Oxalis corniculata L. (black circles), Oxalis cratensis Hook. (white squares), Oxalis divaricata Mart. ex Zucc. (black squares), Oxalis frutescens L. (black triangles) and Oxalis glaucescens Norlind (white triangles). BA: Bahia State. PE: Pernambuco State. SE: Sergipe State.

\& M.C.S. Mota 6989 (MAC); Chã Preta, Serra Lisa, 29-IV2009, fl. e fr., E.C.O. Chagas \& M.C.S. Mota 3339 (MAC); Maceió, Farol, 27-VIII-2009, fl. e fr., E.C.O. Chagas \& M.C.S. Mota 5105 (MAC).

3. Oxalis cratensis Hook., Icon. Pl. 4: pl. 361. 1841.

Figuras 1, 2 g, 4 b-i

Oxalis cratensis distribui-se pela Bolívia, Brasil e Paraguai. No Brasil pode ser encontrada nas regiões Norte (PA, TO), Nordeste (AL, BA, CE, MA, PB, PE, PI, RN, SE), Sudeste (MG, RJ, SP) e Centro-Oeste (GO, MT, MS) (Lourteig 1994, Abreu \& Fiaschi 2015). Oxalis cratensis destaca-se pelo hábito ereto com ramos glabros, flores com lobos da corola róseos e cápsulas globoso-elipsoides. No Estado de Alagoas ocorre na Mata Atlântica, em ambientes sombreados, bordas de mata e margens de estradas (tabela 1). Espécimes podem ser coletados com flores e frutos durante todo o ano.

Material examinado selecionado: BRASIL. ALAGOAS: Boca da Mata, Fazenda Daniel, 28-III-2009, fl. e fr., E.C.O. Chagas et al. 2436 (MAC); Chã Preta, Serra Lisa, 29-IV-2009, fl. e fr., E.C.O. Chagas \& M.C.S. Mota 3313 (MAC); Fleixeiras, Serra das Águas Belas, 2-XI-2002, bot., W.W. Thomas et al. 13159 (HST, MAC); Mar Vermelho, Fazenda Canadá, 30V-2009, fl. e fr., E.C.O. Chagas et al. 3882 (MAC); Matriz do Camaragibe, Usina Serra D'água, 31-X-2009, fl. e fr., E.C.O. Chagas et al. 6378 (MAC); Murici, Bananeiras, Estação Ecológica de Murici, 21-X-2011, fr., E.C.O. Chagas et al. 11241 (MAC); ibid. Serra do Ouro, caminho para a estação da Embratel, 15-III-2002, fl., R.P. Lyra-Lemos et al. 6293 (MAC); ibid. Serra das águas belas, 29-VI-2002, fl. e fr., R.P. Lyra-Lemos et al. 6960 (MAC); Palmeira dos Índios, Serra das Pias, 20-V-2009, bot., S.P. Gomes s.n. (MAC40028); Pão de açúcar, descida da serra do Fuzil, 10-VI-1981, fl., fr. e bot., D.A. Lima et al. 64 (IPA, RB); Pilar, Fazenda Lamarão, 28-VII-2000, fl. e fr., R.P. Lyra-Lemos et al. 4667 (MAC); ibid. Unidade de produção de gás natural, mata do Lamarão, 6-IV-2002, fl., R.P. Lyra-Lemos 6601 (MAC); Rio Largo, 15-III-2008, fl., R.P. Lyra-lemos et al. 10962 (MAC); São Miguel dos Campos, próximo à jazida da argila da Zebu, 26X-2001, fl., R.P. Lyra-Lemos et al. 5886 (MAC); ibid. área da 
reserva da atol cimentos, 20-III-2003, bot., R.P. Lyra-Lemos 7528 (HUEFS, MAC); São Sebastião, Fazenda Indiana, 04X-2009, fl. e fr., E.C.O. Chagas et al. 6115 (MAC); Teotônio Vilela, Reserva Madeiras, 31-VIII-2012, fl. e fr., R.C. Pinto et al. 229 (MAC); Viçosa, Fazenda Jussara, 23-IX-2010, fl. e fr., E.C.O. Chagas et al. 8654 (MAC).

4. Oxalis divaricata Mart. ex Zucc., Denkscr. Konigl. Akad. Wiss. Munchen Ser. 1. 9: 169. 1825.

Figuras 1, 4 a-f

Espécie endêmica do Brasil, com ocorrência nas regiões Nordeste (AL, BA, CE, MA, PB, PE, PI, RN, SE), CentroOeste (GO) e Sudeste (MG, RJ) (Abreu \& Fiaschi 2015). Esta espécie destaca-se pelo hábito herbáceo ou subarbustivo com ramos e sépalas recobertos pro tricomas glandulares, flores com corola amarela e cápsula globoso-elipsoide com até 5 $\mathrm{mm}$ de comprimento. Oxalis divaricata pode ser confundida com $O$. frutescens pela coloração amarela de suas flores. No entanto, diferenciam-se pela disposição alterna ou suboposta das folhas e pelos folíolos com margem inteira, presentes em $O$. divaricata. No Estado de Alagoas é encontrada na Zona das Caatingas e em área de Mata Atlântica (tabela 1). Espécimes foram coletados com flores e frutos entre janeiro e julho.

Material examinado selecionado: BRASIL. ALAGOAS: Água Branca, Morro do Padre, 22-VII-2014, fl., M.W. TavaresSilva et al. 105 (MAC); Cacimbinhas, Serra do Cruzeiro, 30-I-2010, fl., E.C.O. Chagas \& M.C.S. Mota 7364 (MAC); Coité do Noia, Serra do Brejo, 23-III-1983, fl. e fr., M.N.R. Staviski et al. 534 (MAC); Inhapi, Serra do Grude, 23-IV2009, fl., E.C.O. Chagas et al. 3057 (MAC); Jaramataia, próximo ao Povoado Bengo, 25-V-2007, fl., R.P. LyraLemos et al. 10233 (MAC); Olho d'Água do Casado, Fazenda Capelinha, 28-VI-2000, fl. e fr., R.P. Lyra-Lemos 4662 (HST, MAC, RB); Palmeira dos Índios, Serra do Goiti, 22-IV-2000, fl., R.P. Lyra-Lemos et al. 4546 (MAC); Pão de Açúcar, $3 \mathrm{~km}$ depois da zona urbana, na direção da Ilha do Ferro, 15-VII-2000, fl. e fr., R.P. Lyra-Lemos 4835 (MAC); ibid. Boqueirão, 23-II-2002, fl., R.P. LyraLemos et al. 6181 (MAC); Piaçabuçu, Juquinha, 26-VII1988, fl. e fr., G.L. Esteves et al. 2128 (MAC); Piranhas, Povoado de Entremontes, 26-V-2007, fl., R.P. Lyra-Lemos et al. 10457 (MAC); Santana do Ipanema, Serra do Gugi, 31-I-2010, fl., E.C.O. Chagas \& M.C.S. Mota 7472 (MAC); Taquarana, Serra da Itapaiuna, 06-II-2010, fl. e fr., E.C.O. Chagas \& M.C.S. Mota 7586 (MAC).

5. Oxalis frutescens L., Sp. Pl. 1: 435. 1753.

Figuras 1, $4 \mathrm{~g}$

Esta espécie distribui-se desde o México e Antilhas até o Noroeste da Argentina, em Cerrados, Campos e Floresta úmida (Lourteig 1994). No Brasil, Oxalis frutescens ocorre nas regiões Norte (AC, AM, AP, RR, TO), Nordeste (AL, BA, CE, MA, PB, PE, PI, SE), Centro-Oeste (MS, MT) e Sudeste
(ES, MG, RJ) (Abreu \& Fiaschi 2015). Oxalis frutescens é reconhecida pelos braquiblastos, folíolos com formato elíptico e com margem ciliada, presença de uma bráctea por flor e corola com pétalas uniformemente amarelas e cápsulas com 2-4 sementes por lóculo. No Estado de Alagoas, é encontrada na zona das Caatingas, em diferentes fisionomias (tabela 1). Espécimes foram coletados com flores em abril e julho e com frutos em julho.

Material examinado selecionado: BRASIL. ALAGOAS: Mata Grande, Serra Verde, 30-VII-1981, fl. e fr., R.P. Lyra-Lemos et al. 695 (MAC); Olho D'água do Casado, Fazenda Capelinha, 3-VII-2000, fl., R.A. Silva et al. 1532 (RB, SP); Palmeira dos Índios, caminho para a barragem do Riacho Balsamo, 04-IV-2008, fl., R.P. Lyra-Lemos et al. 11115 (MAC).

6. Oxalis glaucescens Norlind, Ark. Bot. 14: 15-16, pl.4, f. 2. 1915.

Figuras 1, $2 \mathrm{~d}, 4 \mathrm{~h}$

Espécie endêmica do Brasil, com ocorrência nas regiões Nordeste (AL, BA, CE, PB, PE, PI, RN, SE), CentroOeste (MS, MT) e Sudeste (MG) (Abreu \& Fiaschi 2015). Oxalis glaucescens apresenta hábito prostrado com folhas trifolioladas, similar a $O$. corniculata, mas são diferenciadas pelas folhas pinadas e cápsulas globoso-achatadas, presentes em O. glaucescens. No Estado de Alagoas, essa espécie ocorre exclusivamente em região de Caatinga (tabela 1). Espécimes foram coletados com flores e frutos em fevereiro, abril e julho.

Material examinado selecionado: BRASIL. ALAGOAs: Água Branca, Fazenda Cobras, 24-IV-2009, fl. e fr., E.C.O. Chagas \& M.C.S. Mota 3099 (MAC); Belo Monte, $6 \mathrm{~km}$ da zona urbana, área da MIBASA, 10-II-2002, fl. e fr., R.P. Lemos et al. 6033 (MAC); Pão de Açúcar, $3 \mathrm{~km}$ depois da zona urbana, na direção da ilha do ferro, 15-VII-2000, fl. e fr., R.P. Lyra-Lemos 4814 (MAC), ibid. Fazenda Morro Preto, 23-II-2002, fl., R.P. Lyra-Lemos et al. 6223 (ESA).

7. Oxalis hedysarifolia Raddi, Mem. Mat. Fis. Soc. Ital. Sci. Moderna, Pt. Mem. Fis. 18: 401. 1820.

Figuras $2 \mathrm{f}, 3,4 \mathrm{c}$

Oxalis hedysarifolia distribui-se na Bolívia, Brasil e Paraguai, em florestas úmidas, cerrados e restingas (Lourteig 1994). No Brasil, está amplamente distribuída nas regiões Norte (PA, RO), Nordeste (AL, BA, CE, MA, PB, PE, PI, SE), Centro-Oeste (GO, MT), Sudeste (ES, MG, RJ, SP) e Sul (SC), ocorrendo nas florestas atlântica e amazônica e nos cerrados (Abreu \& Fiaschi 2015). Esta espécie é reconhecida pelo porte arbustivo com ramos tomentosos e folíolos trulados com ápice agudo e pelas cápsulas oblongas com três sementes por lóculo. No Estado de Alagoas, $O$. hedysarifolia ocorre na subzona do Agreste em região de Caatinga, sobre serras, em campo rupestre e em região de borda e interior de Mata Atlântica (tabela 1). Espécimes foram coletados com flores e frutos entre fevereiro e agosto. 


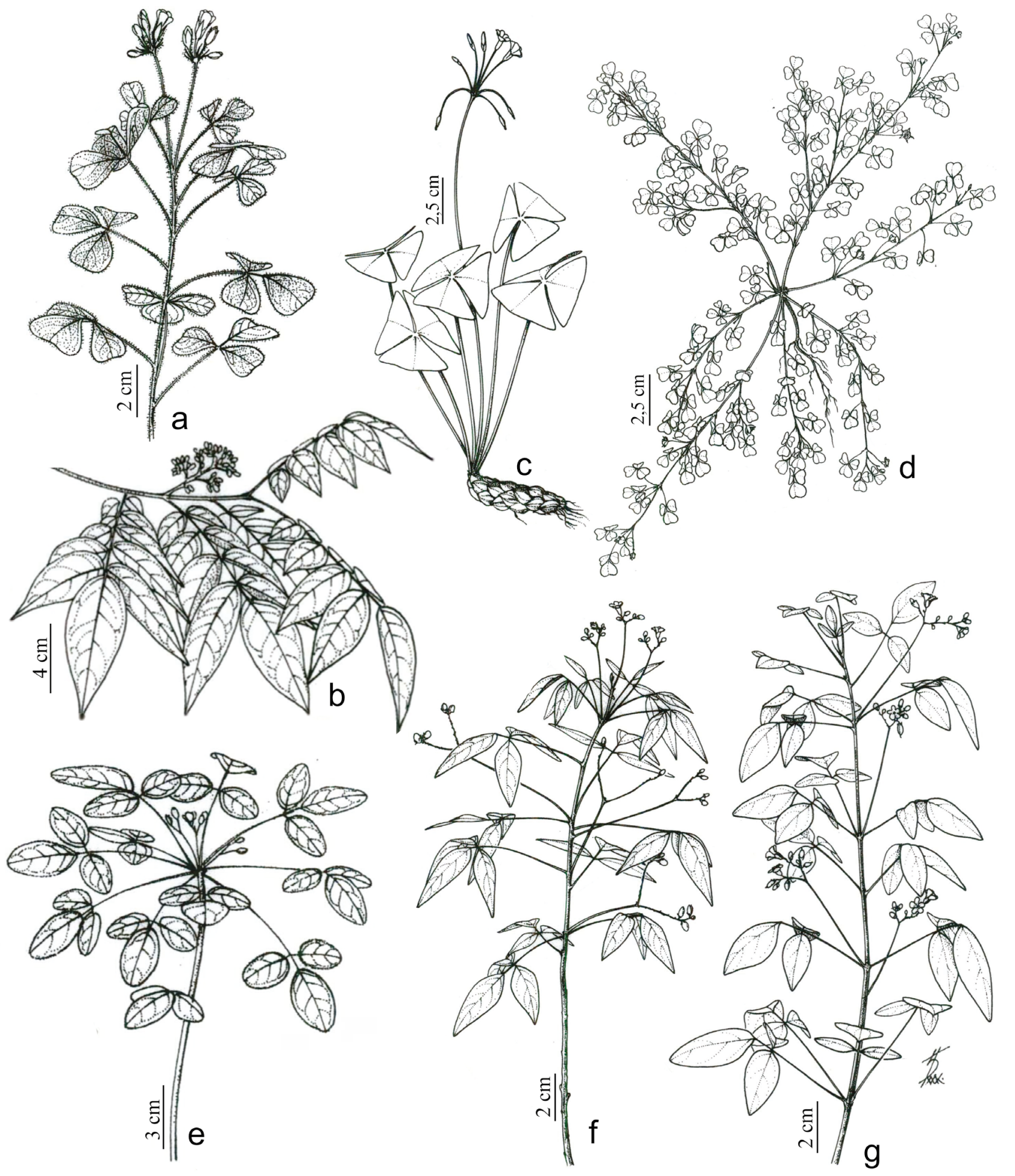

Figura 2. a. Oxalis sepium A. St.-Hil., ramo florido. b. Averrhoa carambola L., ramo florido. c. Oxalis triangularis A. St.-Hil., hábito. d. Oxalis glaucescens Norlind, hábito. e. Oxalis umbraticola A. St.-Hil., ramo florido. f. Oxalis hedysarifolia Raddi, ramo florido. g. Oxalis cratensis Hook., ramo florido.

Figure 2. a. Oxalis sepium A. St.-Hil., branch with flowers. b. Averrhoa carambola L., branch with flowers. c. Oxalis triangularis A. St.-Hil., habit. d. Oxalis glaucescens Norlind, habit. e. Oxalis umbraticola A. St.-Hil., branch with flowers. f. Oxalis hedysarifolia Raddi, branch with flowers. g. Oxalis cratensis Hook, branch with flowers. 


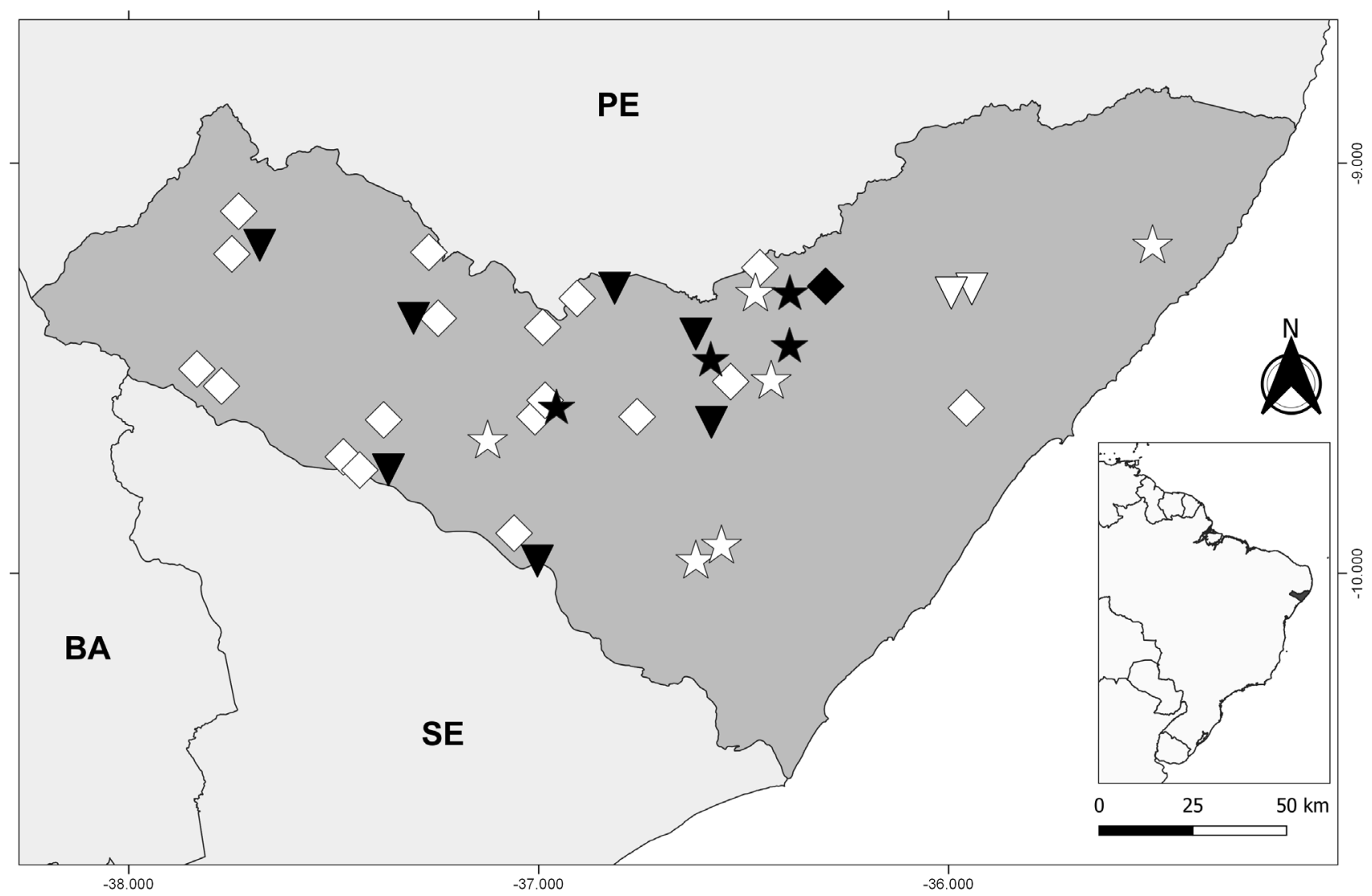

Figura 3. Distribuição das espécies no Estado de Alagoas, Brasil. Oxalis hedysarifolia Raddi (estrelas brancas), Oxalis neuwiedii Zucc. (estrelas pretas), Oxalis psoraleoides Knuth (trapézios brancos), Oxalis sepium A. St.-Hil. (triângulos pretos), Oxalis triangularis A. St.-Hil. (trapézio preto) e Oxalis umbraticola A. St.-Hil. (triângulos brancos). BA: Estado da Bahia. PE: Estado de Pernambuco. SE: Estado de Sergipe.

Figure 3. Distribution of species in Alagoas State, Brazil. Oxalis hedysarifolia Raddi (white stars), Oxalis neuwiedii Zucc. (black stars), Oxalis psoraleoides Knuth (white trapezes), Oxalis sepium A. St.-Hil. (black triangles), Oxalis triangularis A. St.-Hil. (black trapeze) and Oxalis umbraticola A. St.-Hil. (white triangles) BA: Bahia State. PE: Pernambuco State. SE: Sergipe State.

Material examinado selecionado: BRASIL. AlagoAs: Batalha, margens do Rio Traipu, 21-II-2009, fl. e fr., E.C.O. Chagas \& M.C.S. Mota 2100 (MAC); Coruripe, Fazenda Capiatã, 4-V-2005, fl., R.P. Lyra-Lemos 8597 (MAC); Matriz do Camaragibe, Fazenda Brejinho, $8 \mathrm{~km}$ da Usina Camaragibe, 30-VIII-2003, fl., R.P. Lyra-Lemos et al. 7937 (MAC); Palmeira dos Índios, Fazenda Fortaleza, 16-VIII-2001, fl., R.P. Lyra-Lemos et al. 5771 (MAC, RB); ibid. 16.VII.2001, fl. e fr., R.P. Lyra-Lemos et al. 5779 (MAC); ibid. Ponte do rio Alegre, 17-VIII-2006, fl., R.P. Lyra-Lemos 9761 (MAC); Quebrangulo, Reserva Biológica de Pedra Talhada, 11-VI-2011, fl., R.P. Lyra-lemos et al. 13308 (MAC); São Sebastião, Fazenda Indiana, 06-III2010, fl. e fr., E.C.O. Chagas \& M.C.S. Mota 7726 (MAC); Tanque d'Arca, Morro do Cruzeiro, 15-VIII-2009, fl. e fr., E.C.O. Chagas \& M.C.S. Mota 4864 (MAC); Traipu, 21VIII-2010, fl., R.P. Lyra-Lemos et al. 13223 (MAC).
8. Oxalis neuwiedii Zucc., Denkschr. Königl. Akad. Wiss. München 1: 267. 1831.

\section{Figura 3}

Espécie restrita ao território brasileiro (Lourteig 1994), ocorrente nas regiões Nordeste (BA, SE) e Sudeste (ES, RJ) (Abreu \& Fiaschi 2015). Neste estudo é apontada a primeira ocorrência de Oxalis neuwiedii para o Estado de Alagoas. Esta espécie é caracterizada pelo hábito ereto com folhas trifolioladas pseudoverticiladas, flores com lobos da corola alvos e fauces amarelas e cápsulas subglobosas, com uma semente por lóculo. Assemelha-se a Oxalis umbraticola pelo hábito ereto e pela disposição das folhas no ápice dos ramos, mas são diferenciadas pela ausência de indumento nos folíolos, folíolo terminal estreitamente ovado a lanceolado e cápsula globosa. No Estado de Alagoas ocorre em Mata Pluvial e Floresta Ombrófila (tabela 1). Espécimes foram coletados com flores entre maio e outubro e com frutos em outubro. 

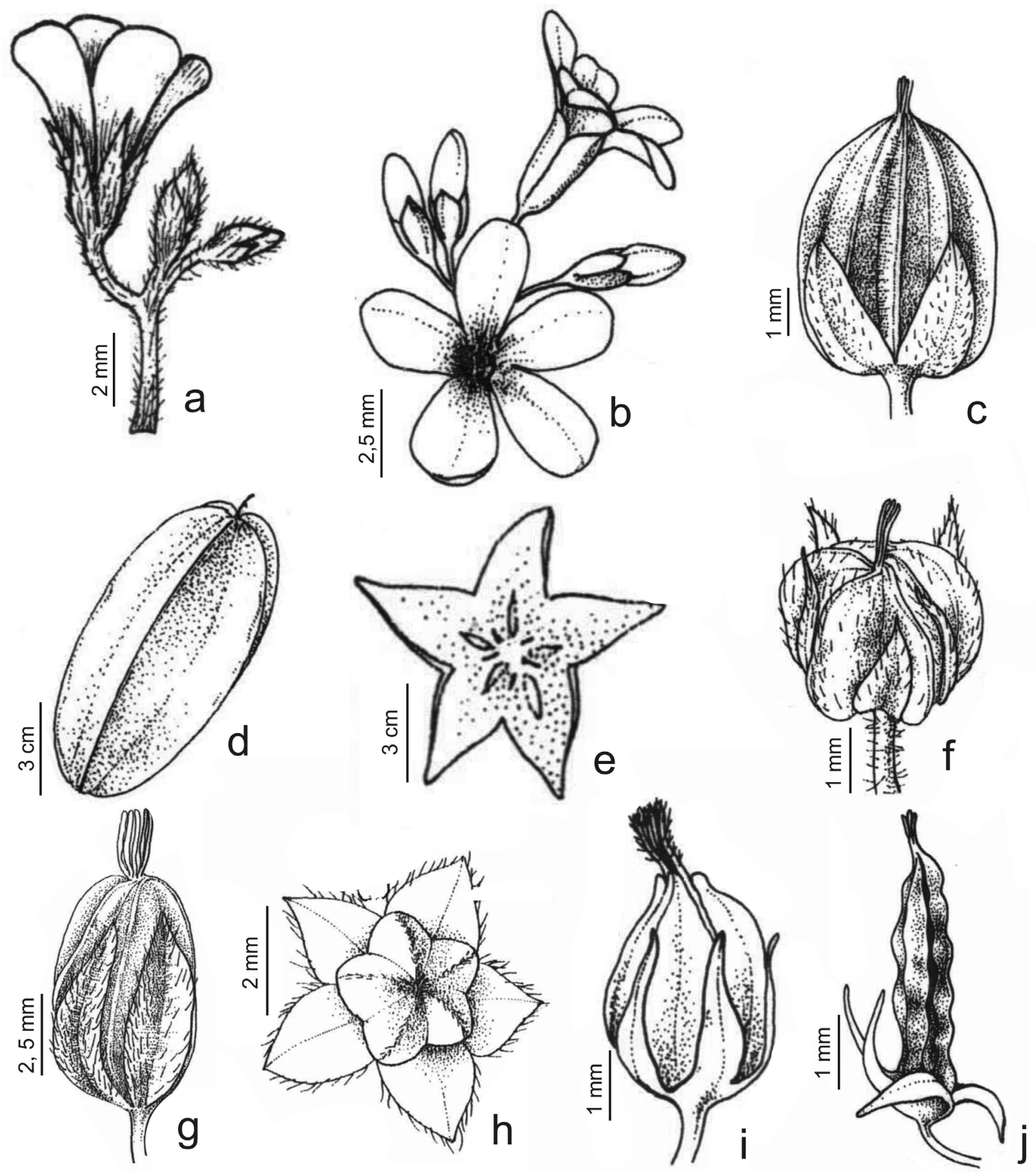

Figura 4. a. Oxalis divaricata Mart. ex Zucc., flor em vista lateral. b. Oxalis cratensis Hook., flores em vista frontal e lateral. c. Oxalis hedysarifolia Raddi, fruto. d-e. Averrhoa carambola L., d. fruto, vista lateral. e. fruto, corte transversal. f. Oxalis divaricata Mart ex Zucc., fruto. g. Oxalis frutescens L., fruto. h. Oxalis glaucescens Norlind, fruto. i. Oxalis cratensis Hook., fruto. j. Oxalis corniculata L., fruto.

Figure 4. a. Oxalis divaricata Mart ex Zucc., flower, lateral view. b. Oxalis cratensis Hook., flowers, frontal and lateral view. c. Oxalis hedysarifolia Raddi, fruit. d-e. Averrhoa carambola L., d. fruit, lateral view. e. fruit, transversal section. f. Oxalis divaricata Mart ex Zucc, fruit. g. Oxalis frutescens L., fruit. h. Oxalis glaucescens Norlind, fruit. i. Oxalis cratensis Hook., fruit. j. Oxalis corniculata L., fruit. 
Material examinado selecionado: BRASIL. ALAGOAS: Chã Preta, RPPN Vera Cruz, 31-VIII-2013, fl., R.L. Silva \& J.S. Correia 63 (MAC); Mar Vermelho, Fazenda Canadá, 08-V2009, fl., E.C.O. Chagas et al. 3584 (MAC); Pilar, Mata das Marrecas, 25-IX-2008, fl., M.N. Rodrigues \& I.A. Bayma 2265 (MAC); Quebrangulo, Reserva Biológica Federal da Pedra Talhada, 31-X-2014, fl. e fr., L. Nusbaumer \& A. Studer 4278 (UFP).

9. Oxalis psoraleoides Kunth, Nov. Gen. Sp. Pl. 5: 246. 1821. Figura 3

Esta espécie ocorre na Bolívia, no Norte do Paraguai, Noroeste da Argentina e Brasil nas regiões Nordeste (AL, BA, CE, PB, PE, PI, RN, SE), Sudeste (MG) e CentroOeste (MS), preferencialmente em locais de elevada altitude (Lourteig 1994, Abreu \& Fiaschi 2015). Oxalis psoraleoides destaca-se pelo hábito arbustivo, com ramos e folhas indumentadas, flores com lobos da corola amarelos e cápsula ovoide-globosa. Entre as espécies listadas no Estado de Alagoas, assemelha-se a Oxalis hedysarifolia, pois ambas possuem caule aéreo e ereto com folhas trifolioladas e flores com pétalas amarelas. Entretanto, O. psoraleoides possui folíolos elípticos com nervuras secundárias proeminentes na face abaxial e cápsulas com uma semente por lóculo, enquanto $O$. hedysarifolia possui folíolos trulados com nervuras secundárias pouco evidentes em ambas as faces e cápsulas com três sementes por lóculo. Segundo Lourteig (1994), há duas subespécies de Oxalis psoraleoides, que são diferenciadas pelo formato dos folíolos e indumento dos folíolos e das sépalas. Entretanto, no Brasil apenas O. psoraleoides subsp. insipida é encontrada, enquanto O. psoraleoides subsp. psoraleoides ocorre na Colômbia, Equador e Peru.

No Estado de Alagoas, O. psoraleoides ocorre em área de Caatinga, em diferentes fitofisionomias desse bioma (tabela 1). Espécimes podem ser coletados com flores e frutos durante todo o ano.

Material examinado selecionado: BRASIL. ALAGOAS: Água Branca, Morro do Craunã, 24-IV-2009, fl. e fr., E.C.O. Chagas et al. 3168 (MAC); Batalha, 21-II-2009, fl., R.P. Lyra-Lemos et al. 11802 (MAC); Cacimbinha, Serra do Cruzeiro, 30-I-2010, fl., E.C.O. Chagas \& M.C.S. Mota 7365 (MAC); Estrela de Alagoas, Fazenda Caititu, 18-I2001, fl. e fr., R.P. Lyra-Lemos 5333 (IPA, MAC); ibid. próximo à BR-316, 18-I-2001, fl. e fr., R.P. Lyra-Lemos 5386 (ESA, MAC); Igaci, Serra do Urubu, 12-XII-2014, f1., R.P. Lyra-Lemos \& J.W. Alves-Silva 13967 (MAC); Inhapi, Serra do Grude, 23-IV-2009, fl. e fr, E.C.O. Chagas \& M.C.S. Mota et al. 2966 (MAC); Jaramataia, Serra das Mãos, 15-XI-2000, fl. e fr., R.P. Lyra-Lemos 5116 (MAC, RB); Maravilha, Serra da Caiçara, 13-V-1982, fl. e fr., R.P. Lyra-Lemos et al. 547 (MAC); ibid. 16-IX-2000, fl. e fr., R.P. Lyra-Lemos 5053 (HUEFS, MAC, RB); ibid. Serra da Caiçara, Fazenda do Dr. Adeval, 23-II-2018, fl., A.P.N.
Prata et al. 3967 (MAC); Mata Grande, Serra Verde, 30VII-1981, fr., R.P. Lyra-Lemos et al. 694 (MAC); ibid. Serra da Boa Vista, 2-VI-2001, fl. e fr., R.P. Lyra-Lemos 5670 (MAC); Olho D'Água do Casado, Fazenda Alagoas, 23-XI-1999, fl., D. Moura 980 (UFP); Palmeira dos Índios, ponte do rio Alegre, 17-VIII-2006, fl. e fr., R.P. Lyra-Lemos 9632 (MAC); ibid. 17-VIII-2006, fl. e fr., R.P. Lyra-Lemos 9274 (MAC); Pão de Açúcar, Serra de Pão de Açúcar, 10VI-1981, fl., R.P. Lyra-Lemos et al. 185 (MAC); ibid. a $5 \mathrm{~km}$ de Pão de Açúcar AL-130, 14-V-1982, fl. e fr., M.N.R. Staviski et al. 457 (MAC); ibid. Riacho Grande, 27-VI-2004, fl., R.P. Lyra-Lemos et al. 8340 (MAC); ibid. Rosa Cruz, 26-X-1982, fl., R.P. Lyra-Lemos et al. 807 (MAC); ibid. próximo ao povoado Meirus, no topo da Serra do Jerônimo, 16-VII-2000, fl. e fr., R.P. Lyra-Lemos 4868 (MAC); Pilar, Lamarão, 07-XI-2002, bot. e fl., G.B. Araújo 128 (MAC); Piranhas, Distrito de Poço Verde, 17-V-1999, fl., R.A. Silva et al. 318 (PEUFR); ibid. Fazenda Baixa da Légua, 27-VI-2000, fl. e fr., I.A. Bayma 403 (MAC); ibid. 27-VI-2000, fl. e fr., R.P. Lyra-Lemos 4656 (ESA, MAC); Quebrangulo, Reserva Biológica Pedra Talhada, 27-II-2010, fl., W.W. Thomas et al. 15196 (MAC); São José da Tapera, 14-VII-2000, fl. e fr., R.P. Lyra-Lemos 4730 (IPA, MAC); Santana do Ipanema, Serra da Camonga, 24-VII-2008, fl., E.C.O. Chagas \& M.C.S. Mota 823 (MAC); ibid. Serra da Micro-ondas, 13V-1982, fl. e fr., R.P. Lyra-Lemos et al. 522 (MAC); Traipu, Serra das Mãos, 22-III-1983, f1. e fr., G.L. Esteves et al. 2149 (MAC); ibid. 15-V-1990, fl. e fr., R.P. Lyra-Lemos et al. 2521 (MAC).

10. Oxalis sepium A. St.-Hil., Fl. Bras. Mer. 1: 111. 1825. Figuras 2 a, 3

Espécie restrita ao Brasil e distribui-se nas regiões Norte (AC, PA, TO), Nordeste (AL, BA, PI, SE), Centro-Oeste (DF, GO, MS, MT) e Sudeste (MG, RJ, SP) (Abreu \& Fiaschi 2015). Oxalis sepium é reconhecida pelo conjunto dos caracteres: erva ou subarbusto com ramos glabros e com folíolos ovados a elípticos, corola com lobos amarelos e cápsulas oblongas. Esta espécie assemelha-se a $O$. divaricata pelo hábito ereto e pela cor da corola e são diferenciadas pela presença de ramos híspidos com tricomas glandulares, folíolos estreitamente ovado-oblongos a lineares e cápsulas globoso-elipsoides, presentes em $O$. divaricata. No Estado de Alagoas, O. sepium ocorre em região de Caatinga e em Brejo de Altitude (tabela 1). Espécimes foram coletados com flores entre maio e setembro e com frutos em junho.

Material examinado selecionado: BRASIL. ALAGOAS: Coité do Noia, Pereiro Velho, 20-VIII-2010, fl., E.C.O. Chagas \& M.C.S. Mota 8173 (MAC); Inhapi, Serra do Grude, 08-VIII-2009, fl., E.C.O. Chagas \& M.C.S. Mota 4658 (MAC); Minador do Negrão, Pedra Talhada, 19-VIII2011, fl., E.C.O. Chagas et al. 11039 (MAC); Palmeira dos Índios, Serra das Pias, 09-V-2009, bot., S.P. Gomes \& D.S. Correia s.n. (MAC37214); Pão de Açúcar, Serra do Fuzil, 
10-VI-1981, fr., G.L. Esteves et al. 680 (MAC); Santana do Ipanema, Serra do Gugi, 10-VII-2009, fl., E.C.O. Chagas et al. 4310 (MAC); Traipu, 22-IX-2007, fl., R.P. Lyra-Lemos et al. 10771 (MAC); ibid. Serra das Mãos, 14-IX-2010, fl., E.C.O. Chagas \& M.C.S. Mota 8567 (MAC).

11. Oxalis triangularis A. St.-Hil., Fl. Bras. Mer. 1:102. 1825.

Figuras 2 c, 3

Espécie encontrada na Bolívia, Paraguai, Brasil e Nordeste da Argentina em campos e terrenos úmidos de baixa altitude (Lourteig 2000). No Brasil, ocorre nas regiões Norte (PA), Nordeste (BA, CE, PB, PE, RN), Centro-Oeste (GO, MS, MT), Sudeste (ES, MG, RJ, SP) e Sul (PR, RS, SC) (Abreu \& Fiaschi 2015). Oxalis triangularis possui hábito herbáceo com bulbos rizomatosos, folhas trifolioladas, sépalas com calosidades no ápice e corola com lobos lilases. A coloração lilás presente na corola é uma característica presente apenas em $O$. triangularis, dentre todas as demais espécies de Oxalis encontradas no Estado de Alagoas. Tratase do primeiro registro dessa espécie para o Estado, sendo coletada nos municípios de Serra Grande (cultivada) e em Murici, em região de Mata Atlântica (tabela 1). Espécimes foram coletados com flores em fevereiro e setembro.

Material examinado selecionado: BRASIL. ALAGOAS: Murici, 13-II-2009, fl., A.I.L. Pinheiro et al. 570 (MAC); Serra Grande, 10-IX-1935, fl., J.G. Sobrinho s.n. (IPA6665).

12. Oxalis umbraticola A. St.-Hil., Fl. Bras. Mer. 1: 111112.1825.

Figuras 2 e, 3

Espécie endêmica do Brasil, que ocorre em áreas úmidas e sombreadas (Lourteig 1994) e distribui-se nas regiões Norte (TO), Nordeste (AL, BA, PE), Centro-Oeste (DF, GO, MT) e Sudeste (ES, MG, RJ, SP) (Abreu \& Fiaschi 2015). Oxalis umbraticola destaca-se das demais espécies encontradas no Estado de Alagoas pelo conjunto dos seguintes caracteres: folhas dispostas em pseudoverticilos, folíolo terminal romboelíptico a rombo-ovado, flores com lobos da corola amarelos e cápsula elipsoide a ovoide. Essa espécie possui apenas um registro para o Estado de Alagoas, coletado em região de Mata Atlântica, no município de Chã Preta (tabela 1). Espécimes foram coletados com flores em outubro.

Material examinado selecionado: BRASIL. ALAGOAS: Chã Preta, Serra Lisa, 16-X-2010, fl., E.C.O. Chagas et al. 9066 (MAC).

\section{Agradecimentos}

Agradecemos aos Curadores e técnicos dos Herbários ESA, HST, HUEFS, IPA, PEUFR, RB e SP, por disponibilizarem os espécimes para este estudo, em especial à
Dra. Rosângela Pereira de Lyra Lemos, Curadora do Herbário MAC. Agradecemos também aos ilustradores Franck Silva e Regina Carvalho, pela confecção das pranchas.

\section{Literatura citada}

Abreu, M.C., Carvalho, R. \& Sales, M.F. 2008. Oxalis L. (Oxalidaceae) no estado de Pernambuco, Brasil. Acta Botanica Brasilica 22: 399-416.

Abreu, M.C. \& Fiaschi, P. 2009. Oxalidaceae. In: Stehmann, J.R., Forzza, R.C., Sobral, M., Salino, A. \& Kamino, L.H.Y. (eds.). Plantas de floresta Atlântica. Jardim Botânico do Rio de Janeiro. Disponível em: http:/www.icb.ufmg.br.bot.mataatlântica (acesso em 13-X-2016).

Abreu, M.C. \& Fiaschi, P. Oxalidaceae. autoria in Flora do Brasil 2020. Jardim Botânico do Rio de Janeiro. Disponível em <http://floradobrasil.jbrj.gov.br/2010/ FB000181> (acesso em 25-III-2019).

Abreu, M.C., Santos, L. L., Chagas, M.G.S. \& Sales, M.F. 2013. Oxalidaceae. In: Rizzo, J.A. Flora dos Estados de Goiás e Tocantins, Goiânia: Gráfica UFG.

Angiosperm Phylogeny Group (APG). 2016. An update of the Angiosperm Phylogeny Group classification for the orders and families of flowering plants: APG IV. Botanical Journal of the Linnean Society 181: 1-20.

Brummitt, R.F. \& Powell, C.E. 1992. Authors of plant names. Royal Botanic Gardens-Kew, Londres.

Cocucci, A.A. 2004. Oxalidaceae. In: Kubitzki, K, (ed.). The families and genera of vascular plants. Springer Verlag, Berlin, Heidelberg.

Conceição, A.A. \& Giulietti, A.M. 1998. Flora da Serra do Cipó, Minas Gerais: Oxalidaceae. Boletim de Botânica da Universidade de São Paulo 17: 115-122.

Conceição, A.A. \& Giullietti, A.M. 2004. Flora de GrãoMogol, Minas Gerais: Oxalidaceae. Boletim de Botânica da Universidade de São Paulo 22: 385-387.

Donadio, L.C., Silva, J.A.A., Araújo, P.S.R. \& Prado, R.M. 2001. Caramboleira (Averrhoa carambola L.). Sociedade Brasileira de Fruticultura.

Fiaschi, P. 2006. Oxalidaceae. In: Cavalcanti, T.B. (eds.). Flora do Distrito Federal, Brasil. Brasília, Distrito Federal.

Fiaschi, P. 2014. Oxalidaceae. In: Kaehler, M., Goldenberg, R., Evangelista, P.H.L., Ribas, O.S., Vieira, A.O.S. \& Hatschbach. G.G. (eds.). Plantas vasculares do Paraná. Universidade Federal do Paraná, Curitiba.

Fiaschi, P. 2018. Check-list da Família Oxalidaceae no estado do Mato Grosso do Sul. Inheringia, Série Botânica 73: 297-300.

Fiaschi, P. \& Conceição, A. A. 2005. Oxalidaceae. In: Wanderley, M.G.L., Shepherd, G.J., Melhem, T.S. \& Giulietti, A. M. (eds.). Flora Fanerogâmica do Estado de São Paulo. São Paulo. 
Grigoletto, D., Bertuzzi, T., Eisinger, S.M., CantoDorow, T.S. \& Colusso, C.S. 2014. O gênero Oxalis L. (Oxalidaceae) no Rio Grande do Sul, Brasil. Ciência e Natura 36: 594-612.

Groom, Q., Van Der Straeten, J., Hoste, I. 2019. The origin of Oxalis corniculata L. Peerj 7: e6384.

Hall, C.F., Meirelles, J. \& Fiaschi, P. 2017. Flora das cangas da Serra dos Carajás, Pará, Brasil: Oxalidaceae. Rodriguésia 68: 1067-1071.

Harris, J.G. \& Harris, M.W. 1994. Plant identification terminology. An illustrated glossary. Spring Lake.

IMA. 2005. Estado de Alagoas. Disponível em http: // www. ima.al.gov.br (acesso em 13-IV-2009).

Judd, W.S., Campbell, C.S., Kellogg, E.A. \& Stevens, P.F. 1999. Plant Systematics: A phylogenetic approach. Massachusetts, Sinauer Associates.

Loiola, M.I.B., Ribeiro, R.T.M., Leles, F.A.M., Tabosa, F.R.S. \& Abreu, M.C. 2018. Flora do Ceará, Brasil: Oxalidaceae. Rodriguésia 69: 863-880.

Lourteig, A. 1975. Oxalidaceae extra-austroamericanae I. Oxalis L. Sectio Thamnoxys Planchon. Phytologia 29: 449- 471.

Lourteig, A. 1979. Oxalidaceae extra-austroamericanae II. Oxalis L. Sectio Corniculatae DC. Phytologia 42: 57-198.

Lourteig, A. 1980. Oxalidaceae extra-austroamericanae III. Oxalis L. Subgenus Monoxalis Lourteig. Phytologia 46: 451-459.

Lourteig, A. 1982. Oxalidaceae extra-austroamericanae IV. Oxalis L. Sectio Articulatae Knuth. Phytologia 50: 130-142.

Lourteig, A. 1983. Oxalidáceas. In: Reitz, R. Flora Ilustrada Catarinense, parte 1, fascículo Oxal. Itajaí, Santa Catarina.
Lourteig, A. 1994. Oxalis L. Subgênero Thamnoxys (Endl.) Reiche emend. Lourt. Bradea 7: 1-199.

Lourteig, A. 2000. Oxalis L. Subgéneros Monoxalis (Small) Lourt., Oxalis y Trifidus Lourt. Bradea 7: 202-629.

Lyra-Lemos, R.P., Mota, M.C.S., Chagas, E.C.O. \& Silva, F.C. 2010. Checklist Flora de Alagoas: Angiospermas. Imperador.

Melo, J.I.M. \& Lyra-Lemos, R.P. 2008. Sinopse taxonômica de Boraginaceae sensu lato A. Juss. no Estado de Alagoas, Brasil. Acta Botanica Brasilica 22: 701-710.

Oberlander, K.C., Dreyer, L.L. \& Bellstedt, D.U. 2011. Molecular phylogenetics and origins of southern African Oxalis. Taxon 60: 1667-1677.

Progel, A. 1877. Oxalideae. In: Von Martius, C.F.P. \& Eichler, A.G. (eds.) Flora Brasiliensis 12: 473-520.

QGIS. GRASS-PROJECT. 2013. Geographic resource analysis support system. Disponível em: http:/grass. osgeo.org.

Radford, A.E., Dickson, W.C., Massey, J.R. \& Bell. C. R. 1974. Vascular Plant Systematics. New York, Happer and Row.

Souza, F.O. \& Bianchini, R.S. 2000. Oxalidaceae. Flora Fanerogâmica do Parque Estadual das Fontes do Ipiranga (São Paulo, Brasil). Hoehnea, 27:117-120.

Souza, V.C. \& Lorenzi, H. 2008. Botânica sistemática: guia ilustrado para identificação das famílias de fanerógamas nativas e exóticas no Brasil, baseado em APG II. São Paulo: Instituto Plantarum.

Stafleu, F.A. \& Cowan, R.S. 1976-1988. Taxonomic Literature. Utrecht, Scheltema \& Holkema.

Thiers, B. 2019. Index herbariorium: a global directory of public herbaria and associated staff. New York Botanical Garden's Virtual Herbarium. Disponível em http:// sweetgum.nybg.org/science/ih/ (acesso em 29-III-2019). 